\title{
THE SPECTRA AND COMMUTANTS OF SOME WEIGHTED COMPOSITION OPERATORS
}

\author{
JAMES W. CARLSON
}

\begin{abstract}
An operator $T_{u g}$ on a Hilbert space $H$ of functions on a set $X$ defined by $T_{u g}(f)=u(f \circ g)$, where $f$ is in $H, u: X \rightarrow \mathbf{C}$ and $g: X \rightarrow X$, is called a weighted composition operator. In this paper $X$ is the set of integers and $H=L^{2}(\mathbf{Z}, \mu)$, where $\mu$ is a measure whose sigma-algebra is the power set of $\mathbf{Z}$. One distinguished space is $l^{2}=L^{2}(\mathbf{Z}, \mu)$, where $\mu$ is counting measure. The most important results given here are the determination of the spectrum of $T_{u g}$ on $l^{2}$ and a characterization of the commutant of $T_{g}$ on $L^{2}(\mathbf{Z}, \mu)$. To obtain many of the results it was necessary to assume the function $g$ to be one-to-one except on a finite subset of the integers.
\end{abstract}

\section{INTRODUCTION}

If $H$ is a Hilbert space of complex valued functions on a set $X$, an operator $A$ on $H$ is called a composition operator if there is a function $g: X \rightarrow X$ such that $A f=f \circ g$ for all $f$ in $H$. Composition operators that may be found in the literature are substitution operators, translation operators, and pointwise induced operators. Unilateral shifts, bilateral shifts, and translation on spaces $L^{2}(G)$, where $G$ is a topological group, are examples of composition operators that have been studied extensively. An operator $T$ on $H$ is called a weighted composition operator if there is a complex valued function $u$ on $X$ and a function $g: X \rightarrow X$ such that $T f=(u)(f \circ g)$ for all $f$ in $H$. Weighted shifts on $l^{2}$ are examples of well-studied weighted composition operators (see Shields [25]). S. K. Parrott, in his thesis [22], studied multiplication by a function $u$ on $H=L^{2}(X, v)$ and composition with an invertible measure preserving transformation $g$ of $H=L^{2}(X, v)$, where $v$ is a sigma-finite measure. In his paper [21], E. Nordgren summarized known information about composition operators on $L^{2}$ and $H^{2}$ spaces. An extensive reference list is also included in Nordgren's paper. After the appearance of Nordgren's paper other authors, such as Kumar, Singh, and Whitley, presented results about composition operators on $L^{2}$ and other authors, such as Cowen, Kamowtiz, Kumar, and Marshall presented results about composition operators on $H^{2}$.

Received by the editors August 13, 1986 and, in revised form, March 20, 1988.

1980 Mathematics Subject Classification (1985 Revision). Primary 47B37.

This paper includes part of the author's dissertation written under Professor Carl Cowen at Purdue University. 
The definition of weighted composition operator given above is too general to hope to obtain many results. In the present work, study has been restricted to the case when $X$ is the set of integers and $H=l^{2}$ is viewed as a Hilbert space of functions on the integers. A few papers that have appeared concerning weighted composition operators in this setting are those of Gupta, Kumar, and Singh [10], Komal and Singh [14], and Singh [28]. Gupta, Kumar, and Singh [10] discuss quasinormal composition operators on a weighted space $l_{p}^{2}$ where the weights are square summable; Komal and Singh [14] give elementary properties of composition operators on $l^{p}$ spaces; Singh [28] provides information about composition operators on weighted spaces $L^{2}(\lambda)$.

Throughout this work $\mathbf{Z}, \mathbf{N}, \mathbf{R}, \mathbf{C}$, and $\mathbf{T}$ denote the sets of integers, positive integers, real numbers, complex numbers and the set of complex numbers of modulus one, respectively. By the word operator we will mean a linear operator but not necessarily a bounded operator. If $T$ is an operator then $\sigma(T)$, $\sigma_{p}(T), \sigma_{e}(T)$, and $\{T\}^{\prime}$ denote the spectrum, point spectrum, essential spectrum, and commutant of $T$. Also, $\|\cdot\|$ and $\langle\cdot, \cdot\rangle$ denote the norm and inner product on $l^{2}$ and, for each $n$ in $\mathbf{Z}, e_{n}$ denotes the function on $\mathbf{Z}$ that is one at $n$ and zero otherwise. Note that $\left\{e_{n}\right\}_{n \in \mathbf{Z}}$ is an orthonormal basis for $l^{2}$.

\section{Preliminaries}

The formal definition of a weighted composition operator used in this work is stated here:

If $T$ is a linear operator on $l^{2}$ defined by

$$
T f(n)= \begin{cases}u(n)(f \circ g) & \text { if } n \text { is in } Y, \\ 0 & \text { otherwise }\end{cases}
$$

for all $f$ in $l^{2}$ and $n$ in $\mathbf{Z}$, where $Y$ is a subset of $\mathbf{Z}, g: Y \rightarrow \mathbf{Z}$ and $u: \mathbf{Z} \rightarrow \mathbf{C}$, then $T$ is called a weighted composition operator on $l^{2}$. In this case we will denote $T$ by $T_{u g}, u$ will be called a weight function, and $g$ will be called a composition function. If $u$ is identically equal to one then the notation $T_{g}$ may be used for $T_{u g}$.

If $n \geq 0$ then the $n$ th-iterate of $g$ is given by $g^{(n)}=g \circ g \circ \cdots \circ g$, $g$ composed with itself $n$-times. If $n>0$ and $k$ is in $\mathbf{Z}$ then $g^{-n}(k)=$ $\left\{j\right.$ in $\left.\mathbf{Z}: g^{(n)}(j)=k\right\}$. In the case when $g^{-n}(k)$ is a singleton, $g^{-n}(k)=\{j\}$, there will be no distinction made between $j$ and $\{j\}$.

It will be helpful to find formulas for $T_{u g}$ and $T_{u g}^{*}$. Let $f=\sum_{n \in Z} f_{n} e_{n}$ be in $l^{2}, Y$ be a subset of $\mathbf{Z}, g: Y \rightarrow \mathbf{Z}$ and $u: \mathbf{Z} \rightarrow \mathbf{C}$. Then

$$
T_{u g}(f)=\sum_{n \in Y} f_{g(n)} u(n) e_{n}
$$

and

$$
T_{u g}^{*}(f)=\sum_{n \in \operatorname{Image} g} \sum_{k \in g^{-1}(n)} f_{k} \overline{u(k)} e_{n}
$$


the sums given here are formal sums that may not converge. In the case $f=e_{n}$ for some $n$ in $\mathbf{Z}$,

$$
\begin{aligned}
& T_{u g} e_{n}= \begin{cases}\sum_{k \in g^{-1}(n)} u(k) e_{k} & \text { if } n \text { is in Image } g, \\
0 & \text { otherwise, }\end{cases} \\
& T_{u g}^{*} e_{n}= \begin{cases}\overline{u(n)} e_{g(n)} & \text { if } n \text { is in } Y, \\
0 & \text { otherwise. }\end{cases}
\end{aligned}
$$

Let $Y$ be a subset of $\mathbf{Z}$ and $g: Y \rightarrow \mathbf{Z}$ be a function. For $n$ in $\mathbf{Z}$ the set $[n]_{g}=\left\{k\right.$ in Z: there exist $j \geq 0$ and $t \geq 0$ such that $\left.g^{(j)}(k)=g^{(t)}(n)\right\}$ is called the orbit of $g$ containing $n$. If $k$ is in $\mathbf{Z}$ and $g^{(j)}(k)=k$ for some $j \geq 1$ then the cycle of $g$ containing $k$ is the set $C=\left\{n\right.$ in $\mathbf{Z}: g^{(t)}(k)=n$ for some $t \geq 0\}$; the length of the cycle $C$ is the least integer $j \geq 1$ such that $g^{(j)}(k)=k$. Note that if $C$ is a cycle of $g$ of length $j$ and if $n$ is in $C$ then $g^{(j)}(n)=n$.

Let $Y$ be a subset of $\mathbf{Z}$ and $g: Y \rightarrow \mathbf{Z}$. Define the function $[g]:(Y \cup$ Image $g) \rightarrow \mathbf{Z}$ by

(1) $[g](n)=0$ if $n$ is in a cycle of $g$,

(2) $[g]\left(n_{F}\right)=0$, where $n_{F}$ is a fixed element of each orbit $F$ of $g$ not containing a cycle,

(3) $[g](n)+1=[g](g(n))$ if $n$ is in $Y$ and $n$ is not in a cycle of $g$.

For the purposes of this paper the choice of the integers $n_{F}$ is not important since any other choice can be obtained under a reindexing of the integers. The branch of $g$ containing $n$ is a maximal subset $G$ of $[n]_{g}$ such that $n$ is in $G$, $[g]$ restricted to $G \cap Y$ is one-to-one, and $n$ in $G \cap Y$ implies $g(n)$ is in $G$ if $n$ is not a cycle of $g$. The set of all branches of $g$ will be denoted by br $g$. We will now exploit the fact that a branch of $g$ is linearly ordered by the function $[g]$. For a branch $b$ of $g$ let $\operatorname{nl}(b)=\inf [g](b)$ be the negative length of $b$ and let $\operatorname{pl}(b)=\sup [g](b)$ be the positive length of $b$. A branch $b$ of $g$ gives rise to a function from $[g](b)$ into $b$, this function will also be denoted by $b$ and is given by $b(n)=[g]^{-1}(n) \cap b$ for each $n$ in $[g](b)$.

Example 1. Let $u$ be a weight function on $\mathbf{N}$ and let $g$ and $h$ be composition functions given by $g(n)=n-1$ if $n \geq 1$ and $h(n)=n+1$ if $n \geq 0$ (under a bijection from $\mathbf{N}$ onto $\mathbf{Z}, g$ and $h$ can be thought of as functions on $\mathbf{Z}$ ). The operator $T_{u g}$ is a weighted forward shift and $T_{u h}$ is a weighted backward shift.

One way to visualize weighted composition operators is to use "graphs". In Example 1 the graph

$$
\cdots \rightarrow 3 \rightarrow 2 \rightarrow 1 \rightarrow 0
$$

indicates the action of $g$ on $\mathbf{N}$. The graph

$$
e_{0} \rightarrow e_{1} \rightarrow e_{2} \rightarrow e_{3} \rightarrow \cdots
$$


indicates the action of $T_{u g}$ on the basis $\left\{e_{n}\right\}_{n \geq 0}$. Note that an arrow denotes one basis element being sent into a multiple of the next.

Example 2. If $g: \mathbf{Z} \rightarrow \mathbf{Z}$ is defined by $g(n)=n+1$ when $n \geq 0$ and $g(n)=$ $n+2$ when $n<0$ then $g$ is visualized by the graph

$$
\begin{aligned}
& \cdots \rightarrow-4 \rightarrow-2 \rightarrow 0 \rightarrow 1 \rightarrow 2 \rightarrow 3 \rightarrow \cdots \\
& \cdots \rightarrow-5 \rightarrow-3 \rightarrow-1 .
\end{aligned}
$$

The function $g$ has two branches that may be visualized as

$$
\cdots \rightarrow-4 \rightarrow-2 \rightarrow 0 \rightarrow 1 \rightarrow 2 \rightarrow 3 \rightarrow \cdots
$$

and

$$
\cdots \rightarrow-5 \rightarrow-3 \rightarrow-1 \rightarrow 1 \rightarrow 2 \rightarrow 3 \rightarrow \cdots .
$$

The operator $T_{g}$ can be visualized by the graph

$$
\begin{aligned}
& \cdots \rightarrow e_{2} \rightarrow e_{1} \rightarrow e_{0} \rightarrow e_{-2} \rightarrow \cdots \\
& e_{-1} \rightarrow e_{-3} \rightarrow \cdots
\end{aligned}
$$

where the arrows from $e_{1}$ imply $e_{1}$ is sent into a linear combination of $e_{0}$ and $e_{-1}$. The operator $T_{g}^{*}$ can be visualized by

$$
\begin{aligned}
& \cdots \rightarrow e_{-2} \rightarrow e_{0} \rightarrow e_{1} \rightarrow e_{2} \rightarrow \cdots \\
& \cdots \rightarrow e_{-3} \rightarrow e_{-1} .
\end{aligned}
$$

Example 3. Let $f(n)=(n-1)(n-2)(n-3)$ for $n$ in $\mathbf{Z}, Y=\{k$ in $\mathbf{Z}: k=$ $f^{(j)}(i)$ for some $j \geq 0$ and $\left.i=1,2,3\right\}$, and $g$ be $f$ restricted to $Y$. The graph for $T_{g}$ is

$$
\begin{aligned}
& \begin{aligned}
\cdots \rightarrow e_{-504} \rightarrow e_{-6} \rightarrow e_{0} \rightarrow e_{1} \\
\searrow
\end{aligned} \\
& e_{3} \text {. }
\end{aligned}
$$

Example 4. This example is motivated by a paper of C. C. Cowen [7]. Let $D$ be the unit disc in $\mathbf{C}$ and $g: D \rightarrow D$ be a nonconstant analytic function. If $z$ is a point in $D$ then $F=\left\{b: g^{(k)}(b)=z\right.$ for some $\left.k>0\right\}$ is a countable set. Index $F$ by the negative integers to obtain $F=\left\{z_{k}\right\}_{k<0}$ and for $k>-1$ let $z_{k}=g^{(k)}(z)$. The function $g$ induces $g^{\sim}: \mathbf{Z} \rightarrow \mathbf{Z}$ by $g^{\sim}(k)=j$ if $g\left(z_{k}\right)=z_{j}$. If $g$ has a Denjoy-Wolff point $a$ in $\mathbf{T}, g^{\prime}(a)=1$ and $G=\{f$ in $H^{2}(D): f\left(z_{k}\right)=0$ for all $k$ in $\left.\mathbf{Z}\right\}$ then $G^{\perp}$ is a nontrivial invariant subspace 
for $T_{g}^{*}$ (see Cowen [7]). On $G^{\perp}$ the operator $T_{g}^{*}$ is similar to a weighted composition operator $T_{u g \sim}$ on $l^{2}$.

To conclude this section we point out that weighted composition operators are related to operator weighted shifts. Let $g: \mathbf{Z} \rightarrow \mathbf{Z}, Y \cup$ Image $g=\mathbf{Z}$, and $u$ be a nonzero weight function. For each $n$ in $\mathbf{Z}$ define $H_{n}$ to be the closed span of $\left\{e_{k}:[g](k)=n\right\}$. The operator $T_{u g}$ maps $H_{n}$ into $H_{n-1}$ for each $n$ in $\mathbf{Z}$.

\section{Spectrum}

In this section we want to find the point spectrum, essential spectrum, and spectrum of a weighted composition operator. We will restrict ourselves to the case when a composition function has finitely many branches. For this case the point spectrum, essential spectrum, and spectrum of a weighted composition operator are found in Theorems 2.1,2.2, and 2.3. We will need only consider composition functions with one orbit, since an orbit induces a reducing subspace to which the restriction of the weighted composition operator is again a weighted composition operator. Also, we will assume that $\mathbf{Z}$ is equal to the orbit of the composition function, since a weighted composition operator restricted to the basis elements associated with integers not in an orbit of the composition function is a zero operator.

Let $Y$ be a subset of $\mathbf{Z}, g: Y \rightarrow \mathbf{Z}$ have one orbit and finitely many branches, $\mathbf{Z}=($ Image $g) \cup Y$, and $u$ be a nonzero weight function. The notation given here will make many of the following statements less cumbersome. If $b$ is a branch of $g$ and $j$ is in $[g](b)$ then define

$$
\begin{aligned}
U_{b} & = \begin{cases}\lim _{n \geq 1} \sup |u(b(j-n)) \cdots u(b(j-1))|^{1 / n} & \text { if } \mathrm{nl}(b)=-\infty, \\
0 & \text { otherwise }\end{cases} \\
L_{b} & = \begin{cases}\lim _{n \geq 1} \inf |u(b(j-n)) \cdots u(b(j-1))|^{1 / n} & \text { if } \mathrm{nl}(b)=-\infty, \\
0 & \text { otherwise },\end{cases} \\
L & = \begin{cases}\lim _{n \geq 1} \inf |u(b(j+1)) \cdots u(b(j+n))|^{1 / n} & \text { if } \mathrm{pl}(b)=+\infty, \\
0 & \text { otherwise },\end{cases} \\
U & = \begin{cases}\lim _{n \geq 1} \sup |u(b(j+1)) \cdots u(b(j+n))|^{1 / n} & \text { if } \operatorname{pl}(b)=+\infty, \\
0 & \text { otherwise }\end{cases}
\end{aligned}
$$

The numbers $U_{b}$ and $L_{b}$ do not depend on the choice of $j$ in $[g](b)$. The numbers $L$ and $U$ do not depend on $b$ or $j$ in $[g](b)$.

In Theorem 2.1 we find that the point spectrum of $T_{u g}$ and $T_{u g}^{*}$, when $g$ does not have a cycle, is either a disk, an annulus, or a disk union an annulus. The particular structure of the spectrum depends on the values $U_{b}, L_{b}, U$ and $L$ for all branches $b$ of $g$. In Theorem 2.2 we find that the point spectrum of $T_{u g}$, when $g$ has a cycle, is a finite set of points and that the point spectrum of $T_{u g}^{*}$ is a finite set of points union a disk. When $g$ does not have a cycle 
the spectrum of $T_{u g}$ has circular symmetry since $T_{u g}$ is unitarily equivalent to $T_{|u| g}$ (Carlson [1]).

The results obtained here were foreshadowed by the known structure of the spectrum of a weighted shift on $l^{2}$ (Ridge [23], Shields [25]). A weighted shift is merely a weighted composition operator with one branch $b$ where the spectrum is either an annulus or a disk determined by the values $U_{b}$ and $U$. The question answered here is 'what happens to the spectrum when weighted shifts are pasted together'. With the assumption that the composition operator has only a finite number of branches the results on weighed shifts can be generalized to weighted composition operators by analyzing the point spectrum of the operator and its adjoint.

Theorem 2.1. Let $Y$ be a subset of $\mathbf{Z}, g: Y \rightarrow Z$ have one orbit, finitely many branches, and no cycle, $\mathbf{Z}=Y \cup$ Image $g, u$ be a nonzero weight function, and $T_{u g}$ be a bounded operator. Then

$$
B_{1} \cup\left(\text { Interior of } K_{1}\right) \subset \sigma_{p}\left(T_{u g}\right) \subset B_{1} \cup K_{1}
$$

and

$$
B_{2} \cup \text { Interior of }\left(K_{2} \cup K_{3}\right) \subset \sigma_{p}\left(T_{u g}^{*}\right) \subset B_{2} \cup K_{2} \cup K_{3} \text {, }
$$

where $K_{1}=\left\{z\right.$ in $\mathbf{C}: U_{a} \leq|z| \leq L$ for all $a$ in br $\left.g\right\}, K_{2}=\{z$ in $\mathbf{C}: U \leq$ $|z| \leq L_{a}$ for some $a$ in br $\left.g\right\}, K_{3}=\left\{z\right.$ in $\mathrm{C}:|z| \leq L_{a} \leq L_{b}$ for some $a$ and $b$ in br $g$ such that $a \neq b\}, B_{1}$ is $\{0\}$ if $\mathbf{Z} \backslash$ Image $g$ is not empty and is empty otherwise, and $B_{2}$ is $\{0\}$ if either $\mathbf{Z} \backslash Y$ is not empty or $g$ is not one-to-one and is empty otherwise.

Proof. Let $f=\sum_{n \in \mathrm{Z}} f_{n} e_{n}$ be in $l^{2}$ and $z$ be in $\mathbf{C}$.

$$
\begin{aligned}
\left(T_{u g}-z I\right) f & =\sum_{n \in Y} f_{g(n)} u(n) e_{n}-\sum_{n \in \mathbf{Z}} z f_{n} e_{n} \\
& =\sum_{n \in Y}\left(f_{g(n)} u(n)-z f_{n}\right) e_{n}+\sum_{n \in \mathbf{Z} \backslash Y} z f_{n} e_{n}
\end{aligned}
$$

and

$$
\begin{aligned}
\left(T_{u g}^{*}-z I\right) f & =\sum_{n \in \operatorname{Image} g} \sum_{k \in g^{-1}(n)} f_{k} \overline{u(k)} e_{n}-\sum_{n \in \mathbf{Z}} z f_{n} e_{n} \\
& =\sum_{n \in \text { Image } g}\left(-z f_{n}+\sum_{k \in g^{-1}(n)} f_{k} \overline{u(k)}\right) e_{n}-\sum_{n \in \mathbf{Z} \backslash \text { Image } g} z f_{n} e_{n} .
\end{aligned}
$$

Let $j=\min [g]\left(\bigcap_{a \in \operatorname{br} g} a\right)$ when $g$ has more than one branch and let $j$ be an element of $[g](\mathbf{Z})$ when $g$ has only one branch.

Suppose zero is in the point spectrum of $T_{u g}$; then there is an $f=\sum_{n \in \mathbf{Z}} f_{n} e_{n}$ in $l^{2}$ such that $f$ is not zero and $T_{u g} f=0$. So (2.1) implies $f_{g(n)} u(n)=0$ for all $n$ in $Y$. Thus, $f_{m}=0$ for all $m$ in Image $g$, implying $Z \backslash$ Image $g$ is not empty. 
If $\mathbf{Z} \backslash$ Image $g$ is not empty let $n$ be in $\mathbf{Z} \backslash$ Image $g$; then $T_{u g} e_{n}=0$. Thus, if $\mathbf{Z} \backslash$ Image $g$ is not empty then 0 is in the point spectrum of $T_{u g}$. Therefore 0 is in the point spectrum of $T_{u g}$ if and only if $\mathbf{Z} \backslash$ Image $g$ is not empty. Hence, $B_{1} \subset \sigma_{p}\left(T_{u g}\right)$.

Let $z$ in $\mathbf{C}$ be nonzero and $f=\sum_{n \in \mathbf{Z}} f_{n} e_{n}$ in $l^{2}$ not be zero such that $\left(T_{u g}-z I\right) f=0$. From (2.1) we have $f_{g(n)} u(n)-z f_{n}=0$ for all $n$ in $Y$ and $f_{n}=0$ for all $n$ in $\mathbf{Z} \backslash Y$. Since $z \neq 0, u$ is nonzero, and $\mathbf{Z}=$ Image $g \cup Y$, $f_{n}=0$ if and only if $f_{g(n)}=0$ for all $n$ in $Y$. So, either $f_{n}=0$ for all $n$ in $\mathbf{Z}$ or $f_{n} \neq 0$ for all $n$ in $\mathbf{Z}$. Hence, $\mathbf{Z} \backslash Y$ is empty since $f$ is nonzero. So, $\operatorname{pl}(a)=+\infty$ for all $a$ in $\operatorname{br} g$. Recall that for a branch $a$ of $g$ we denote an element of $a$ by $a(n)$ for some $n$ in $\mathbf{Z}$, so for each branch $a$ of $g$ we have $f_{a(n+1)} u(n)-z f_{a(n)}=0$ for $n$ in $[g](a)$. Now, for $a$ in br $g$ and $m \geq \operatorname{nl}(a)$, if $0 \leq n$ then

$$
f_{a(n+m+1)}=z^{n+1}(u(a(m)) \cdots u(a(n+m)))^{-1} f_{a(m)} \neq 0,
$$

and if $\operatorname{nl}(a) \leq m-n \leq m$ then

$$
f_{a(m-n)}=z^{-n} u(a(m-n)) \cdots u(a(m-1)) f_{a(m)} \neq 0 .
$$

For $a$ in br $g$ and $m \geq \operatorname{nl}(a)$ the sequences

$$
\left\{z^{n+1}(u(a(m)) \cdots u(a(n+m)))^{-1}\right\}_{n \geq 0}
$$

and

$$
\left\{z^{j-m} u(a(j)) \cdots u(a(m-1))\right\}_{\mathrm{nl}(a) \leq j \leq m+1}
$$

are square summable. Thus, the nonzero elements of the point spectrum of $T_{u g}$ are contained in $K_{1}$.

Let $z$ be in interior of $K_{1}$ and $z \neq 0$. If $Z \backslash Y$ is not empty then $L=0$ and Interior of $K_{1}$ is empty. Thus, $\mathrm{Z} \backslash Y$ is empty and $\mathrm{pl}(a)=+\infty$ for each $a$ in br $g$. We wish to find $f$ in $l^{2}$ such that $\left(T_{u g}-z I\right) f=0$. Let $f=$ $\sum_{n \in \mathbf{Z}} f_{n} e_{n}$ where $f_{n}=1$ for $[g](n)=j, f_{n}=u(n) \cdots u\left(g^{(j-m-1)}(n)\right) z^{m-j}$ for $m=[g](n)<j$, and $f_{n}=\left(u\left(g^{(m-j-1)}(i)\right) \cdots u(i)\right)^{-1} z^{m-j}$ for $[g](i)=j$ and $m=[g](n)>j$. Clearly, $f \neq 0$. For $n$ in $\mathbf{Z}$ such that $[g](n)=m$, if $a$ is a branch containing $n$ then $g^{(t)}(n)=a(m+t)$ for $t \geq 0$. Thus, for $n$ in $\mathbf{Z}$ and $n$ contained in the branch $a$, if $m=[g](n)<j$ then

$$
f_{n}=z^{m-j} u(a(m)) \cdots u(a(j-1)),
$$

and if $m=[g](n)>j$ then $f_{n}=z^{m-j}(u(a(j)) \cdots u(a(m-1)))^{-1}$. The sequence $\left\{f_{n}\right\}_{n \in Z}$ is square summable since $g$ has finitely many branches and $z$ 
is in $K_{1}$. Hence $f$ is in $l^{2}$. Now

$$
\begin{aligned}
\left(T_{u g}-z I\right) f= & \sum_{n \in Y}\left(f_{g(n)} u(n)-z f_{n}\right) e_{n} \\
= & \sum_{\substack{n \in Y \\
m=[g](n)<j-1}}\left(u(n) u(g(n)) \cdots u\left(g^{(j-m-1)}(n)\right) z^{m-j+1}\right. \\
& +\sum_{\substack{n \in Y \\
m=[g](n)=j-1}}\left(u(n)-z u(n) z^{-1}\right)+\left(u(i)(u(i))^{-1} z-z\right) \\
& +\sum_{\substack{n \in Y \\
m=[g](n)>j}}\left(u(i) \cdots u\left(g^{(m-j)}(i)\right)\right)^{-1} u\left(g^{(m-j)}(i)\right) z^{m+j+1} \\
= & \left.-z\left(u(i) \cdots u\left(g^{(m-j-1)}(i)\right)\right)^{-1} z^{m-j}\right)
\end{aligned}
$$

where $[g](i)=j$. Thus, $f$ is an eigenvector for $T_{u g}$ with eigenvalue $z$. The set Interior of $K_{1}$ is contained in the point spectrum of $T_{u g}$.

Suppose that zero is in the point spectrum of $T_{u g}^{*}$. There is an $f=\sum_{n \in \mathbf{Z}} f_{n} e_{n}$ in $l^{2}$ such that $f \neq 0$ and $T_{u g}^{*} f=0$. By (2.2) $\sum_{k \in g^{-1}(n)} f_{k} u(k)=0$ for all $n$ in Image $g$. If $g$ is one-to-one and $\mathbf{Z}=Y$ then $f_{k} \overline{u(k)}=0$ for all $k$ in $\mathbf{Z}=Y$. Thus, $g$ is not one-to-one or $\mathbf{Z} \backslash Y$ is not empty.

Suppose $g$ is not one-to-one. There are $k_{1}$ and $k_{2}$ in $Y$ such that $k_{1} \neq k_{2}$ and $g\left(k_{1}\right)=g\left(k_{2}\right)$. The function $f=\overline{u\left(k_{2}\right)} e_{k_{1}}-\overline{u\left(k_{1}\right)} e_{k_{2}}$ is in $l^{2}$ and $T_{u g}^{*} f=$ 0 .

Suppose $\mathbf{Z} \backslash Y$ is not empty. There is an $n$ in $\mathbf{Z}$ such that $n$ is not in $Y$. Now $T_{u g}^{*} e_{n}=0$ and zero is in the point spectrum of $T_{u g}^{*}$. Thus, $B_{2} \subset \sigma_{p}\left(T_{u g}^{*}\right)$.

Let $z$ in $\mathbf{C}$ be nonzero and $f$ in $l^{2}$ be such that $f \neq 0$ and $\left(T_{u g}^{*}-z I\right) f=0$. By (2.2), $z f_{n}-\sum_{k \in g^{-1}(n)} f_{k} \overline{u(k)}=0$ for all $n$ in Image $g$ and $z f_{n}=0$ for all $n$ in $\mathbf{Z} \backslash$ Image $g$. Either there is a branch $a$ of $g$ and $K>0$ such that $\operatorname{nl}(a)=-\infty, \operatorname{pl}(a)=+\infty$,

$$
f_{a(-n-K)}=z^{n}(\overline{u(a(-n-K)) \cdots u(a(-K-1))})^{-1} f_{a(-K)} \neq 0
$$

and

$$
f_{a(n+K)}=z^{-n} \overline{u(a(K)) \cdots u(a(n+K-1))} f_{a(-K)} \neq 0
$$

for all $n \geq K$ or there are two distinct branches $a$ and $b$ of $g$ and $K>0$ such that $\operatorname{nl}(a)=-\infty, \operatorname{nl}(b)=-\infty$,

$$
0 \neq f_{a(-n-K)}=z^{n}(\overline{u(a(-n-K)) \cdots u(a(-K-1))})^{-1} f_{a(-K)},
$$

and

$$
0 \neq f_{b(-n-K)}=z^{n}(\overline{u(b(-n-K)) \cdots u(b(-K-1))})^{-1} f_{b(-K)}
$$

for all $n>0$. 
So, either there is a branch $a$ such that $\operatorname{pl}(a)=+\infty, \operatorname{nl}(a)=-\infty$, and $\left\{z^{-n} u(a(j)) \cdots u(a(n+j-1))\right\}_{n \geq 1}$ and $\left\{z^{n}(\overline{u(a(-n+j)) \cdots u(a(-1+j))})^{-1}\right\}_{n \geq 1}$ are square summable or there are two distinct branches $a$ and $b$ such that $\operatorname{nl}(a)=-\infty, \operatorname{nl}(b)=-\infty$, and

$$
\left\{z^{n}(\overline{u(a(-n+j)) \cdots u(a(-1+j))})^{-1}\right\}_{n \geq 1}
$$

and

$$
\left\{z^{n}\left(\overline{u(b(j)) \cdots u(b(n+j-1)))}^{-1}\right\}_{n \geq 1}\right.
$$

are square summable. Thus, $z$ is contained in $K_{2} \cup K_{3}$.

Let $z$ be in Interior of $K_{2}$ (note that $z \neq 0$ ). There is a branch $a$ of $g$ such that $U<|z|<L_{a}$. We will now find an $f$ in $l^{2}$ such that $\left(T_{u g}^{*}-z I\right) f=0$. Define $f=\sum_{n \in \mathbf{Z}} f_{a(n)} e_{a(n)}$, where

$$
f_{a(j)}=1, \quad f_{a(n+j)}=z^{-n} \overline{u(a(j)) \cdots u(a(n+j-1))} \text { for } n \geq 1,
$$

and

$$
f_{a(j-n)}=z^{n}(u(a(j-n)) \cdots u(a(j-1)))^{-1} \quad \text { for } n \geq 1 .
$$

Clearly, $f \neq 0$ and $f$ is in $l^{2}$. Furthermore,

$$
\left(T_{u g}^{*}-z I\right) f=\sum_{n \in \mathbf{Z}}\left(-z f_{a(n)}+f_{a(n-1)} \overline{u(a(n-1))}\right) e_{a(n)}=0 .
$$

Thus, $f$ is an eigenvector of $T_{u g}^{*}$ for the eigenvalue $z$.

Let $z$ be in Interior of $K_{3}$ such that $z \neq 0$. There are two distinct branches $a$ and $b$ of $g$ such that $z<L_{a} \leq L_{b}$. Define $f=\sum_{n \leq 1} f_{a(n)} e_{a(n)}+f_{b(n)} e_{b(n)}$ where $1+i=\min [g](a \cap b), f_{a(i)}=\overline{u(b(i))}, f_{b(i)}=-\overline{u(a(i))}$, and $f_{a(i-n)}=$ $\overline{u(b(i))} z^{n}(\overline{u(a(i-n)) \cdots u(a(i-1))})^{-1}$ for $n \geq 1$. The function $f$ is in $l^{2}, f$ is not zero, and $\left(T_{u g}^{*}-z I\right) f=0$. Thus, $f$ is an eigenvector of $T_{u g}^{*}$ for the eigenvalue $z$.

Theorem 2.2. Let $g: \mathbf{Z} \rightarrow \mathbf{Z}$ have one orbit, finitely many branches, and exactly one cycle $C$ of length $c$, let $u$ be a nonzero weight function, and let $T_{u g}$ be a bounded operator. Then $B \cup K_{1} \subset \sigma_{p}\left(T_{u g}\right) \subset K_{2} \cup B$ and $K_{3} \cup\{0\} \subset \sigma_{p}\left(T_{u g}^{*}\right) \subset$ $K_{4} \cup\{0\}$, where $K_{1}=\left\{z\right.$ in $\mathbf{C}: z^{c}=u(n) \cdots u\left(g^{(c-1)}(n)\right)$ for $n$ in $C$ and $|z|>U_{a}$ for all $a$ in br $\left.g\right\}, K_{2}=\left\{z\right.$ in $\mathbf{C}: z^{c}=u(n) \cdots u\left(g^{(c-1)}(n)\right)$ for $n$ in $C$ and $|z| \geq U_{a}$ for all $a$ in br $\left.g\right\}, K_{3}=\left\{z \in \mathbf{C}: z^{c}=u(n) \cdots u\left(g^{(c-1)}(n)\right)\right.$ for $n$ in $C$ or $|z|<L_{a} \leq L_{b}$ for some $a$ and $b$ in br $g$ such that $\left.a \neq b\right\}$, $K_{4}=\left\{z\right.$ in $\mathbf{C}: z^{c}=u(n) \cdots u\left(g^{(c-1)}(n)\right)$ for $n$ in $C$ or $|z| \leq L_{a} \leq L_{b}$ for some $a$ and $b$ in br $g$ such that $a \neq b\}$, and $B$ is $\{0\}$ when $\mathrm{Z} \backslash$ Image $g$ is not empty or $B$ is empty otherwise.

Proof. If $f=\sum_{n \in \mathbf{Z}} f_{n} e_{n}$ is in $l^{2}$ and $z$ is in $C$ then the formulas (2.1) and (2.2) still hold. 
Suppose $f=\sum_{n \in \mathrm{Z}} f_{n} e_{n}$ in $l^{2}$ is not zero and $T_{u g} f=0$. By (2.1), $f_{n} \neq$ 0 for some $n$ in $\mathbf{Z} \backslash$ Image $g$. Thus, if zero is an eigenvalue for $T_{u g}$ then $\mathbf{Z} \backslash$ Image $g$ is not empty.

Suppose there is an $n$ in $\mathbf{Z} \backslash$ Image $g$. Then $T_{u g} e_{n}=0$. Thus, if $\mathbf{Z} \backslash$ Image $g$ is not empty then zero is an eigenvalue of $T_{u g}$. Therefore zero is in the point spectrum of $T_{u g}$ if and only if $\mathbf{Z} \backslash$ Image $g$ is not empty.

Suppose $z$ in $\mathbf{C}$ is not zero and $f=\sum_{n \in \mathbf{Z}} f_{n} e_{n}$ in $l^{2}$ is not zero such that $\left(T_{u g}-z I\right) f=0$. From $(2.1)$ we have $f_{g(n)} u(n)-z f_{n}=0$ for all $n$ in $Y$ and $f_{n}=0$ for all $n$ not in $Y$. So

$$
f_{g^{(k)}(n)}=z^{k}\left(u(n) \cdots u\left(g^{(k-1)}(n)\right)\right)^{-1} f_{n}
$$

for $n$ in $C$ and $k \geq 1$ and

$$
f_{a(-k)}=z^{-k} u(a(-k)) \cdots u(a(-1)) f_{a(0)}
$$

for all branches $a$ of $g$ and $1 \leq k \leq|\operatorname{nl}(a)|$. Hence, $z^{c}=u(n) \cdots u\left(g^{(c-1)}(n)\right)$ for $n$ in $C$ and the sequence $\left\{z^{-k} u(a(-k)) \cdots u(a(-1))\right\}_{k=1}^{|n|(a) \mid}$ is square summable for each $a$ in br $g$. Thus, $\sigma_{p}\left(T_{u g}\right) \subset B \cup K_{2}$.

Now suppose $z$ is in $K_{1}$ and $z$ is not zero. Fix $n$ in $C$. It is our task to find $f$ in $l^{2}$ such that $\left(T_{u g}-z I\right) f=0$. Let $f_{n}=1, f_{g^{(k)}(n)}=$ $z^{k}\left(u(n) \cdots u\left(g^{(k-1)}(n)\right)\right)^{-1}$ for $1 \leq k \leq c-1$, and $f_{m}=z^{-k} u(m) \cdots$ $u\left(g^{(k)}(m)\right) f_{g^{(k+1)}(m)}$ for $m$ in $\mathbf{Z}$ where $[g](m)=-k-1$. Define $\sum_{m \in \mathbf{Z}} f_{m} e_{m}=$ $f$. Since $g$ has finitely many branches and $z$ is in $K_{1} \backslash\{0\}, f$ is in $l^{2}$. Now

$$
\left(T_{u g}-z I\right) f=\sum_{m \in \mathbf{Z}}\left(f_{g(m)} u(m)-z f_{m}\right) e_{m}=0 .
$$

Thus, $f$ is an eigenvector of $T_{u g}$ for the eigenvalue $z$. Hence, $K_{1} \subset \sigma_{p}\left(T_{u g}\right)$.

There is a branch $b$ of $g$ such that $\operatorname{nl}(b)=-\infty$, since $g$ has only one orbit and finitely many branches. If

$$
\left.f=\overline{u\left(g^{(c-1)}(b(0))\right.}\right) e_{b(-1)}-u(b(-1)) e_{g^{(c-1)}(b(0))}
$$

then $T_{u g}^{*} f=0$. Thus, 0 is in the point spectrum of $T_{u g}^{*}$.

Let $z$ in $\mathbf{C}$ be nonzero and $f=\sum_{n \in \mathbf{Z}} f_{n} e_{n}$ in $l^{2}$ be such that $f \neq 0$ and $\left(T_{u g}^{*}-z I\right) f=0$. From (2.2) we have $z f_{n}-\sum_{k \in g^{-1}(n)} f_{k} u(k)=0$ for all $n$ in Image $g$ and $f_{n}=0$ for all $n$ not in Image $g$. Either there are two distinct branches $a$ and $b$ of $g$ and $K>0$ such that $\operatorname{nl}(a)=-\infty, \operatorname{nl}(b)=-\infty$,

$$
f_{a(-k)}=z^{k}(u(a(-k)) \cdots u(a(-K-1)))^{-1} f_{a(-K)} \neq 0
$$

and

$$
f_{b(-k)}=z^{k}(u(b(-k)) \cdots u(b(-K-1)))^{-1} f_{b(-K)} \neq 0
$$

for all $k>K$ or

$$
f_{g^{(k)}(n)}=z^{-k} \overline{u(n)} \cdots \overline{u\left(g^{(k-1)}(n)\right)} f_{n} \neq 0
$$


for all $n$ in $\mathbf{C}$ and $k>0$. Thus, either $\left.z^{c}=\overline{u(n)} \cdots \overline{u\left(g^{(c-1)}(n)\right.}\right)$ for $n$ in $\mathbf{C}$ or for two distinct branches $a$ and $b$ of $g$ such that $\operatorname{nl}(a)=-\infty, \operatorname{nl}(b)=$ $-\infty\left\{z^{k}(u(a(-1)) \cdots u(a(-k)))^{-1}\right\}_{k \geq 1}$ and $\left\{z^{k}(u(b(-1)) \cdots u(b(-k)))^{-1}\right\}_{k \geq 1}$ are square summable. Hence, $z$ is in $K_{4}$.

Let $z$ be in $K_{3}$ and $z \neq 0$. If $z^{c}=\overline{u(n)} \cdots \overline{u\left(g^{(c-1)}(n)\right)}$ for $n$ in $\mathbf{C}$ then fix $n$ in $\mathbf{C}$ and let

$$
f=\sum_{0 \leq k \leq c-1} z^{-k} \overline{u(n)} \cdots \overline{u\left(g^{(k-1)}(n)\right)} e_{g^{(k)}(n)}
$$

if $|z|<L_{a} \leq L_{b}$ for two distinct, intersecting branches $a$ and $b$ of $g$ such that $\operatorname{nl}(a)=-\infty$ and $\operatorname{nl}(b)=-\infty$. Then let

$$
\begin{aligned}
h= & \sum_{k>|i|} \overline{u(b(i-1))} z^{k}(\overline{u(a(-k) \cdots u(a(i-2))})^{-1} e_{a(-k)} \\
& -\sum_{k>|i|} \overline{u(a(i-1))} z^{k}(\overline{u(b(-k)) \cdots u(b(i-2))})^{-1} e_{b(-k)},
\end{aligned}
$$

where $i=\min [g](a \cap b)$. If $|z|<L_{a} \leq L_{b}$ for two nonintersecting branches $a$ and $b$ of $g$ such that $\operatorname{nl}(a)=-\infty, \operatorname{nl}(b)=-\infty$, and $b(0)=g^{(t)}(a(0))$ then let

$$
\begin{aligned}
p= & \sum_{k>0} u\left(g^{(t-1)}(a(0))\right) z^{k}(u(b(-k)) \cdots u(b(-2)))^{-1} e_{k} \\
& -\sum_{0 \leq k \leq t-1} u(b(-1)) z^{t-k}\left(u\left(g^{(k)}(a(0))\right) \cdots u\left(g^{(t-2)}(a(0))\right)^{-1} e_{g^{(k)}(a(0))}\right. \\
& -\sum_{k>0} u(b(-1)) z^{t+k}(u(a(-k))) \cdots u(a(0)) \cdots u\left(g^{(t-2)}(a(0))\right)^{-1} e_{a(-k)} .
\end{aligned}
$$

The function $f$ is in $l^{2}$ since $f$ is a finite linear combination of basis elements. The functions $h$ and $p$ are in $l^{2}$ since $|z|<L_{a} \leq L_{b}$. Furthermore, $\left(T_{u g}^{*}-z I\right) f=0,\left(T_{u g}^{*}-z I\right) h=0$, and $\left(T_{u g}^{*}-z I\right) p=0$ Hence, $z$ is in the point spectrum of $T_{u g}^{*}$.

Let $Y$ be a subset of $\mathrm{Z}, g: Y \rightarrow \mathrm{Z}$ have finitely many branches and only one orbit, $\mathbf{Z}=Y \cup \operatorname{Image} g$, and $u$ be a nonzero weight function. The operator $T_{u g}$ is a finite rank perturbation of a finite direct sum of shift operators. We will make this more precise. There is an $i$ in $\mathbf{Z}$ such that if $i$ is in $[g] b$ for some branch $b$ of $g$ then $\operatorname{nl}(b)=-\infty$ and $\min [g](a \cap b)>i$ for any other branch $a$ of $g$. There is a $j$ in $\mathbf{Z}$ such that $[g]^{-1}(n)$ contains at most one element for $n \geq j$.

Let $N$ be the span of $\left\{e_{n}: i<[g](n) \leq j\right\}$ and $F=\left(T_{u g}\right.$ restricted to $\left.N\right)$. Clearly, $F$ has finite rank. Let $W e_{n}=u\left(g^{-1}(n)\right) e_{g^{(-1)}(n)}$ if $[g](n)>j$. For each $a$ in br $g$ such that $\operatorname{nl}(a)=-\infty$ let $W_{a} e_{a(k)}=u(a(k-1)) e_{a(k-1)}$ if $k<i$. The spectra of $W_{a}$ and $W$ are well-studied sets (see Shields [25]). The operator $\left(T_{u g}-F\right)$ is equal to $W \oplus\left(\bigoplus_{a \in \operatorname{br} g, \mathrm{nl}(a)=-\infty} W_{a}\right)$. The following theorem is a consequence of standard results on spectra of compact perturbations. 
Theorem 2.3. Let $Y, g, u, W$, and $W_{a}$ for $a$ in br $g$ be as above. Suppose $T_{u g}$ is a bounded operator. Then

(1) $\sigma_{e}\left(T_{u g}\right)=\sigma_{e}(W) \cup\left(\bigcup_{a \in \operatorname{br} g, \mathrm{nl}(a)=-\infty} \sigma_{e}\left(W_{a}\right)\right)$,

(2) $\sigma\left(T_{u g}\right)=\sigma_{p}\left(T_{u g}\right) \cup \sigma_{p}\left(T_{u g}^{*}\right) \cup \sigma_{e}\left(T_{u g}\right)$.

Example 5. Let $g: \mathbf{Z} \rightarrow \mathbf{Z}$ by

$$
g(n)= \begin{cases}n+1 & \text { if } n \geq-1 \\ n+2 & \text { if } n<-1\end{cases}
$$

and let $u: \mathbf{Z} \rightarrow \mathbf{C}$ by

$$
u(n)= \begin{cases}1 & \text { if } n<-1 \text { and } n=2 k \text { for some } k \text { in } \mathbf{Z}, \\ 2 & \text { if } n<-1 \text { and } n=2 k-1 \text { for some } k \text { in } \mathbf{Z}, \\ 3 & \text { if } n \geq-1 .\end{cases}
$$

The function $g$ has two branches, $a_{1}=\{\ldots,-4,-2,0,1,2, \ldots\}$ and $a_{2}=$ $\{\ldots,-3,-1,0,1,2, \ldots\}$. The compact operator $F$ is given by

$$
F e_{n}= \begin{cases}u(-2) e_{-2}+u(-1) e_{-1} & \text { if } n=0, \\ u(n-2) e_{n-2} & \text { if } n=-1,-2, \\ 0 & \text { otherwise. }\end{cases}
$$

Also,

$$
\begin{aligned}
& W e_{n}=u(n-1) e_{n-1} \quad \text { for } n \geq 1, \\
& W_{a_{1}} e_{n}=u(n-2) e_{n-2} \text { for } n<-2
\end{aligned}
$$

and $n=2 k$ for some $k$ in $\mathbf{Z}$, and

$$
W_{a_{2}} e_{n}=u(n-2) e_{n-2} \text { for } n<-1
$$

and $n=2 k-1$ for some $k$ in $\mathbf{Z}$. Now

$$
\begin{gathered}
\sigma_{p}\left(T_{u g}\right)=\{\mathbf{z} \text { in } \mathbf{C}: 2<|z|<3\}, \quad \sigma_{p}\left(T_{u g}^{*}\right)=\{z \text { in } \mathbf{C}:|z|<1\}, \\
\sigma_{e}(W)=\{z \text { in } \mathbf{C}:|z|=3\}, \quad \sigma_{e}\left(W_{a_{1}}\right)=\{z \text { in } \mathbf{C}:|z|=1\}, \\
\sigma_{e}\left(W_{a_{2}}\right)=\{z \text { in } \mathbf{C}:|z|=2\} .
\end{gathered}
$$

Thus,

$$
\sigma\left(T_{u g}\right)=\{z \text { in } \mathbf{C}:|z| \leq 1\} \cup\{z \text { in } \mathbf{C}: 2 \leq|z| \leq 3\} .
$$

We have seen that the structure of the spectrum of a weighted composition operator on $l^{2}$ is the type of structure we would get by taking direct sums of weighted shifts. Thus, from the perspective of weighted shifts the results given are not surprising. If we change our perspective and recall Example 4 of $\S 1$ and the paper of Cowen [7] then the results seem to be more unexpected. In Example 4, for a weighted composition operator $T_{g}$ on $H^{2}$ of the unit disk there is an associated weighted composition operator $T_{u g} \sim$ on $l^{2}$. Cowen [7] gives some results where $T_{u g}$ is a weighted shift whose spectrum can be an 
annulus but the spectrum of $T_{g}$ is a disk. A natural question to ask is: Can we obtain results about the spectrum of $T_{g}$ knowing the spectrum of $T_{u g \sim}$ ? More particular, are there composition operators on $H^{2}$ of the disk whose spectra are the disjoint union of a disk and an annulus?

The hypothesis that the composition function $g$ has finitely many branches was more than a minor assumption in this section. The results would be different if we were to assume $g$ has infinitely many branches. Shields [25] makes note of an example of an operator with spectrum the unit disk, but the operator is the countably infinite direct sum of weighted shifts all of whose spectra are a single point. One of the difficulties in assuming $g$ has infinitely many branches is that the operator $F$ needed to make $T_{u g}-F$ a direct sum of weighted shifts may no longer be compact. Another difficulty would be in calculating the point spectrum of $T_{u g}$ where the limits involved could move from branch to branch without progressing toward the end of any branch. The structure of $\sigma\left(T_{u g}\right)$ is not clear when $g$ has infinitely many branches.

\section{Commutant}

We wish to characterize the commutant of a weighted composition operator $T_{u g}$ on $l^{2}$. In order to obtain the results given here it is necessary to make some nontrivial restrictions on the composition function $g$. These restrictions are: $g$ is defined on all of $\mathbf{Z}, g$ has exactly one orbit, $g$ does not have a cycle, and all branches of $g$ have the same negative length.

Consider Hilbert spaces of the form $L^{2}(\mathbf{Z}, \mu)$, where $\mu$ is a measure whose sigma-algebra is the power set of $\mathbf{Z}$. If $\mu$ is counting measure $(\mu\{n\}=1$ for all $n$ in $\mathbf{Z}$ ) then $l^{2}=L^{2}(\mathbf{Z}, \mu)$. We are introducing weighted spaces $L^{2}(\mathbf{Z}, \mu)$ because an operator $T_{u g}$ on $l^{2}$ is unitarily equivalent to an operator $T_{g}$ on some weighted space $L^{2}(\mathbf{Z}, \mu)$. The computations are much easier when working with $T_{g}$ on $L^{2}(\mathbf{Z}, \mu)$, hence, we will find the commutant of $T_{g}$ on $L^{2}(\mathbf{Z}, \mu)$ instead of the commutant of $T_{u g}$ on $l^{2}$. The subscript $\mu$ may be used to reference the space $L^{2}(\mathbf{Z}, \mu)$, for example, $\|\cdot\|_{\mu}$ will denote the norm on $L^{2}(\mathbf{Z}, \mu)$.

Let $\mu$ be a measure with sigma-algebra the power set of $\mathbf{Z}$. Then $\left\{e_{n}\right\}_{n \in \mathbf{Z}}$ is an orthogonal basis for $L^{2}(\mathbf{Z}, \mu)$, but $\left\{e_{n}\right\}_{n \in \mathbf{Z}}$ is not necessarily normalized since $\left\|e_{n}\right\|_{\mu}^{2}=\mu(n)$ for $n$ in $\mathbf{Z}$. For $f=\sum_{n \in \mathbf{Z}} f_{n} e_{n},\|f\|_{\mu}^{2}=\sum_{n \in \mathbf{Z}}\left|f_{n}\right|^{2} \mu(n)$. If $A$ is a linear operator on $L^{2}(\mathbf{Z}, \mu)$ then the matrix for $A$ relative to $\left\{e_{n}\right\}_{n \in \mathbf{Z}}$ is given by $\left(A_{i j}\right)_{i, j \in \mathbf{Z}}$, where $\mu(i) A_{i j}=\left\langle A e_{j}, e_{i}\right\rangle_{\mu}$ for $i$ and $j$ in $\mathbf{Z}$. Weighted composition operators on a weighted space $L^{2}(\mathbf{Z}, \mu)$ are defined exactly as they were for the unweighted space $l^{2}$. The adjoint of a weighted composition operator $T_{u g}$ on $L^{2}(\mathbf{Z}, \mu)$ does vary from the $l^{2}$ case; we present the formula 
for $T_{g}^{*}$ acting on $e_{n}, n \in \mathbf{Z}$ :

$$
T_{g}^{*} e_{n}=\left\{\begin{array}{l}
\frac{\mu(n)}{\mu(g(n))} e_{g(n)} \text { for } n \text { in the domain of } g, \\
0 \text { otherwise. }
\end{array}\right.
$$

Proposition 3.1. Let $Y$ be a subset of $\mathbf{Z}, g: Y \rightarrow Z$ have one orbit and no cycle, $\mathbf{Z}=(Y \cup$ Image $g)$, and $u: \mathbf{Z} \rightarrow \mathbf{R}$ be a positive weight function. The operator $T_{u g}$ on $l^{2}$ is unitarily equivalent to an operator $T_{g}$ on $L^{2}(\mathbf{Z}, \mu)$ for some measure $\mu$.

Proof. Define the measure $\mu$ by $\mu(0)=1$ and

$$
\mu(n)=\left[u(0) \cdots u\left(g^{(t-1)}(0)\right)\left[u(n) \cdots u\left(g^{(k-1)}(n)\right)\right]^{-1}\right]^{-2},
$$

where $g^{(k)}(n)=g^{(t)}(0)$ for some $k \geq 0$ and $t \geq 0$. Let $U: l^{2} \rightarrow L^{2}(\mathbf{Z}, \mu)$ by $U f=\mu^{-1 / 2} f$ for $f$ in $l^{2}$. For $f$ in $l^{2}$,

$$
\|U f\|_{\mu}^{2}=\int_{\mathbf{Z}} \mu^{-1}|f|^{2} d \mu=\int_{\mathbf{Z}}|f|^{2} d 1=\|f\|^{2} .
$$

If $f$ is in $l^{2}$ and $h$ is in $L^{2}(\mathbf{Z}, \mu)$ then

$$
\begin{aligned}
\langle U f, h\rangle_{\mu} & =\left\langle\mu^{-1 / 2} f, h\right\rangle_{\mu}=\int_{\mathbf{Z}} \mu^{-1 / 2} f \bar{h} d \mu \\
& =\int_{\mathbf{Z}} f_{\mu}^{1 / 2} \bar{h} d 1=\left\langle f, \mu^{1 / 2} h\right\rangle=\left\langle f, U^{-1} h\right\rangle .
\end{aligned}
$$

Thus, $\|U\|=1$ and $U^{*}=U^{-1}$ implying $U$ is unitary. Let $f$ be in $l^{2}$ and $n$ be in $\mathbf{Z}$. Then

$$
\begin{aligned}
U^{*} T_{g} U f(n) & =U^{*} T_{g}\left(\left(\mu^{-1 / 2} f\right)(n)\right) \\
& =\left\{\begin{array}{l}
U^{*}\left((\mu \circ g)^{-1 / 2}(f \circ g)(n)\right) \text { for } n \text { in } Y, \\
0 \text { otherwise, }
\end{array}\right. \\
& =\left\{\begin{array}{l}
\mu^{1 / 2}(n)(\mu \circ g)^{-1 / 2}(n)(f \circ g)(n) \text { for } n \text { in } Y, \\
0 \text { otherwise, }
\end{array}\right. \\
& =\left\{\begin{array}{r}
\frac{u(0) \cdots u\left(g^{(t-1)}(0)\right)\left[(g(n)) \cdots u\left(g^{(k-2)}(g(n))\right)\right]^{-1}}{\left.u(0) \cdots u\left(g^{(t) 1}\right)(0)\right)\left(u(n) \cdots u\left(g^{(k-1)}(n)\right)\right)^{-1}}(f \circ g)(n) \quad \text { for } n \text { in } Y, \\
\text { where } g^{(k)}(n)=g^{(t)}(0) \text { for some } k \geq 0 \text { and } t>0, \\
0 \text { otherwise, }
\end{array}\right. \\
& = \begin{cases}u(n)(f \circ g)(n) \text { for } n \text { in } Y, \\
0 \text { otherwise, }\end{cases} \\
& =T_{u g} f(n) .
\end{aligned}
$$

Hence, $T_{g}$ on $L^{2}(\mathbf{Z}, \mu)$ is unitarily equivalent to $T_{u g}$ on $l^{2}$.

Some of the material that follows requires us to look at operators that may not be bounded. In order to handle unboundedness we introduce the subspace $N(\mathbf{Z}, \mu)$ of $L^{2}(\mathbf{Z}, \mu)$ consisting of all finite linear combinations of the basis 
elements $\left\{e_{n}\right\}_{n \in \mathbf{Z}}$. If the composition function has a finite number of branches then a weighted composition operator and its adjoint both map $N(\mathbf{Z}, \mu)$ into $N(\mathbf{Z}, \mu)$. Every operator on $L^{2}(\mathbf{Z}, \mu)$ has a natural restriction to an operator from $N(\mathbf{Z}, \mu)$ into $L^{2}(\mathbf{Z}, \mu)$ and every bounded operator on $N(\mathbf{Z}, \mu)$ has a unique extension to an operator on $L^{2}(\mathbf{Z}, \mu)$.

The following proposition will be used repeatedly during the rest of this exposition. It is also a generalization of Proposition 5 from [25].

Proposition 3.2. Let $\mu$ be a positive measure on $\mathbf{Z}$ with sigma-algebra the power set of $\mathbf{Z}$ and let $g: \mathbf{Z} \rightarrow \mathbf{Z}$ be a function. Furthermore, let $A$ be an operator from $N(\mathbf{Z}, \mu)$ into $N(\mathbf{Z}, \mu)$. The equation $A T_{g} e_{n}=T_{g} A e_{n}$ holds for all $n$ in $\mathbf{Z}$ if and only if for all $j$ and $n$ in $\mathbf{Z}$,

$$
A_{g(j), n}=\left\{\begin{array}{l}
\sum_{k \in g^{-1}(n)} A_{j k} \text { if } n \text { is in Image } g, \\
0 \text { otherwise. }
\end{array}\right.
$$

Proof. Let $n$ be in $\mathbf{Z}$. Then

$$
T_{g} A e_{n}=T_{g}\left(\sum_{m \in \mathbf{Z}} A_{m, n} e_{m}\right)=\sum_{j \in \mathbf{Z}} A_{g(j), n} e_{j}
$$

and

$$
\begin{aligned}
A T_{g} e_{n} & =\left\{\begin{array}{l}
A\left(\sum_{k \in g^{-1}(n)} e_{k}\right) \quad \text { if } n \text { is in Image } g, \\
0 \text { otherwise, }
\end{array}\right. \\
& =\left\{\begin{array}{ll}
\sum_{j \in \mathrm{Z}} \sum_{k \in g^{-1}(n)} A_{j k} e_{j} \\
0 \text { otherwise. }
\end{array} \text { if } n \text { is in Image } g,\right.
\end{aligned}
$$

Thus, $T_{g} A e_{n}=A T_{g} e_{n}$ for $n$ in $\mathbf{Z}$ if and only if, for all $j$ and $n$ in $\mathbf{Z}$,

$$
A_{g(j), n}=\left\{\begin{array}{l}
\sum_{k \in g^{-1}(n)} A_{j k} \quad \text { if } n \text { is in Image } g, \\
0 \text { otherwise. }
\end{array}\right.
$$

One case where the commutant of a weighted composition operator is well known is for weighted shifts. If $T_{g}$ is a unilateral shift on a weighted space $L^{2}(\mathbf{Z}, \mu)$ then $T_{g}$ is unitarily equivalent to multiplication by $z$ on a weighted $H^{2}$ space of the unit disc denoted $H^{2}(\beta)$. The commutant of multiplication by $z$ on $H^{2}(\beta)$ is the set of operators that are multiplication by some $h$ in $H^{2}(\beta)$ where $h f$ is in $H^{2}(\beta)$ for all $f$ in $H^{2}(\beta)$ (Shields [25]). A weighted composition operator in general is not multiplication by $z$ on some space. Thus, we cannot hope for the same type of characterization of the commutant of a weighted composition operator that is not a shift. Every $h$ in $H^{2}(\beta)$ is a formal power series in $z$ and so commutes with $z$. We will pursue this idea and try to characterize the commutant of $T_{g}$ on $L^{2}(\mathbf{Z}, \mu)$ as the set of operators $T_{h}$ where $h$ commutes with $g$.

In Proposition 3.3 we show that to determine the structure of an operator $A$ in the commutant of $T_{g}$ on $L^{2}(\mathbf{Z}, \mu)$ it is sufficient to determine the structure of one diagonal of finite dimensional submatrices for the matrix of $A$. 
Theorem 3.4 treats the case when only one of the submatrices of the diagonal of $A$ determined in Proposition 3.3 is nonzero. The results of Theorem 3.4 are extended in Theorem 3.5 where all operators in the commutant of $T_{g}$ are characterized.

Let $g: \mathbf{Z} \rightarrow \mathbf{Z}$ have one orbit, no cycle and finitely many branches, and let $\mu$ be a positive measure on $\mathbf{Z}$. Define $F[g]=\sup \left\{n: g^{-1}(k)\right.$ contains at most one element for all $k$ such that $[g](k) \leq n\}$. For $m$ in $\mathbf{Z}$ and $b$ a branch of $g$ define $S_{m}^{g}=\operatorname{span}\left\{e_{n}:[g](n)=m\right\}$ and

$$
m_{b}=\left\{\begin{array}{l}
\max (F[g]+m, \mathrm{nl} b) \quad \text { if } m<0 \\
\max (F[g], \mathrm{nl} b) \quad \text { if } m \geq 0
\end{array}\right.
$$

Proposition 3.3. Let $A$ and $B$ be operators on $N(\mathbf{Z}, \mu)$ with matrices $\left(A_{i j}\right)_{i, j \in \mathbf{Z}}$ and $\left(B_{i j}\right)_{i, j \in \mathbf{Z}}$. Let $g: \mathbf{Z} \rightarrow \mathbf{Z}$ have more than one branch, finitely many branches, no cycles and one orbit. Furthermore, suppose that $T_{g} A e_{n}=A T_{g} e_{n}$ and $T_{g} B e_{n}=B T_{g} e_{n}$ for all $n \in \mathbf{Z}$. Then $A_{i j}=B_{i j}$ for all $i$ and $j$ in $\mathbf{Z}$ if and only if $A_{b\left(m_{b}\right), \beta\left(m_{b}-m\right)}=B_{b\left(m_{b}\right), \beta\left(m_{b}-m\right)}$ for all $m$ in $\mathbf{Z}$ and $b, \beta \in$ br $g$ such that $\mathrm{nl} \beta \leq m_{b}-m$.

Proof. It is clear that $A_{i j}=B_{i j}$ for all $i$ and $j$ in $\mathbf{Z}$ implies $A_{b\left(m_{b}\right), \beta\left(m_{b}-m\right)}=$ $B_{b\left(m_{b}\right), \beta\left(m_{b}-m\right)}$ for all $m$ in $\mathbf{Z}$ and $b$ and $\beta$ in br $g$ such that $\mathrm{nl} \beta \leq m_{b}-m$.

Conversely, let $i$ and $j$ be in Z . Choose $s=[g](i), t=[g](j)$ and $m=$ $s-t$. If $i=b(s)$ and $s=m_{b}$ for some $b$ in br $g$ then $j=\beta(t)=\beta\left(m_{b}-m\right)$ for some $\beta$ in br $g$ and $A_{i j}=B_{i j}$.

Suppose $i=b(s)$ and $s<m_{b}$ for some $b$ in br $g$. Now $m_{b}-(s-t)=$ $m_{b}-m \leq F[g]$ and, by Proposition 3.2, $B_{h(s+x), \beta(t+x)}=B_{h(s+x+1), \beta(t+x+1)}$ for all $0 \leq x \leq m_{b}-s-1$ and $h, \beta$ in br $g$ such that $h(s+x)$ and $\beta(t+x)$ exist. Thus

$$
B_{i j}=B_{b(s), \beta(t)}=B_{b\left(s+\left(m_{b}-s-1\right)+1\right), \beta\left(t+\left(m_{b}-s-1\right)+1\right)}=B_{b\left(m_{b}\right), \beta\left(m_{b}-m\right)}
$$

where $j=\beta(t)$ for some $\beta$.

Finally, suppose $s>m_{b}$ for all $b$ in $\operatorname{br} g$ such that $i=b(s)$. If $b$ and $\beta$ are in br $g$ such that $i=b(s), j=\beta(t)$ and $\mathrm{nl} \beta \leq m_{b}-m$ then

$$
B_{b\left(s-\left(s-m_{b}\right)\right), \beta\left(t-\left(s-m_{b}\right)\right)}^{\prime}=B_{b\left(m_{b}\right), \beta\left(m_{b}-m\right)^{\prime}} .
$$

If $b$ and $\beta$ are in br $g$ such that $i=b(s), j=\beta(t)$ and $\mathrm{nl} \beta>m_{b}-m$ then

$$
B_{b(s-(t-\mathrm{nl} \beta)), \beta(t-(t-\mathrm{nl} \beta))}=B_{b(s-t+\mathrm{nl} \beta), \beta(\mathrm{nl} \beta)}=0
$$

by Proposition 3.2. 
Now let $b$ and $\beta$ be in br $g$ such that $i=b(s)$ and $j=\beta(t)$, let $0 \leq x<$ $s-m_{b}$ and let $\mathrm{nl} \beta \leq t-x$. Then, by Proposition 3.2,

$$
\begin{aligned}
B_{b(s-x), \beta(t-x)} & =\sum_{y \in g^{-1}(\beta(t-x))} B_{b(s-x-1), y} \\
& =\sum_{\substack{h \in \operatorname{br} g \\
h(t-s)=\beta(t-x) \\
\mathrm{n} l \\
h \leq t-x-1}} \frac{1}{H_{h, t-x-1}} B_{b(s-x-1), h(t-x-1)}
\end{aligned}
$$

(where $H_{h, t-x-1}=$ cardinality $\{a$ in br $g: a(t-x-1)=h(t-x-1)\}$ )

$$
=\sum_{\substack{\beta \in \mathrm{br} g \\ \beta(t)=j \\ \mathrm{nl} \beta \leq m_{b}-m}} \frac{1}{M_{\beta}} B_{b\left(m_{b}\right), \beta\left(m_{b}-m\right)},
$$

where $b$ is in br $g$ with $b(s)=i$ and $M_{\beta}=\prod_{i=m_{b}-m}^{t-x-1} H_{\beta, i}$.

We will now characterize those operators $A$ that commute with $T_{g}$. The first result, Theorem 3.4, addresses the case when $A$ is an operator weighted shift acting on the subspaces $S_{m}^{g}$. Theorem 3.4 characterizes $A$ as a linear combination of operators $T_{x}$ where $x$ commutes with $g$. The second result, Theorem 3.5, extends the result of Theorem 3.4 to all operators that commute with $T_{g}$.

Theorem 3.4. Let $g: \mathbf{Z} \rightarrow \mathbf{Z}$ have one orbit, no cycle, more than one branch, and only a finite number of branches. In addition, assume there is a $K$ in $\mathbf{Z} \cup\{-\infty\}$ such that $\mathrm{nl} b=K$ for all $b$ in $\operatorname{br} g$. On the space $L^{2}(\mathbf{Z}, \mu)$, if $A$ is in the commutant of $T_{g}$ and there is an $m$ in $\mathbf{Z}$ such that $A\left(S_{p}^{g}\right) \subset\left(S_{p+m}^{g}\right)$ for all $p$ in $\mathbf{Z}$ then $A$ restricted to $N(\mathbf{Z}, \mu)$ is in the span of $M=\left\{T_{X}: X \circ g=\right.$ $g \circ X, X: \mathbf{Z} \rightarrow \mathbf{Z}\}$.

Proof. Let br $g=\left\{b_{1}, \ldots, b_{n}\right\}, \mathbf{J}=\{1, \ldots, n\}, m$ be a fixed integer and $A$ be in the commutant of $T_{g}$ such that $A\left(S_{p}^{g}\right) \subset\left(S_{p+m}^{g}\right)$ for all $p$ in $\mathbf{Z}$. The matrix for $A$ with respect to $\left\{e_{n}\right\}$ will be denoted $\left(A_{i j}\right)_{i, j \in \mathbf{Z}}$.

Since all branches of $g$ have the same negative length there is a fixed integer $P=m_{b}$ for all $b$ in $\operatorname{br} g$. We need to show that the matrix for $A$ is in the span of $M$. By Proposition 3.3, we need to show there is a matrix $\left(B_{i j}\right)_{i, j \in \mathbf{Z}}$ in the span of $M$ with $A_{b(P), \beta(P-m)}=B_{b(P), \beta(P-m)}$ for all $b, \beta$ in br $g$ such that $\mathrm{nl} \beta \leq P-m$, since it is clear that $A_{b(s), \beta(t)}=0$ for all $b, \beta$ in br $g$ and $s, t$ in $\mathbf{Z}$ such that $s-t \neq m$. For each $k$ in $\mathbf{Z}$ and $p$ in $\mathbf{J}$ define $c_{p k}=$ $\left\{s\right.$ in $\left.\mathbf{J}: \inf [g]\left(b_{s} \cap b_{p}\right) \leq F[g]+k\right\}$.

Note that if $k \leq 0$ then

$$
\varsigma_{p k}= \begin{cases}\varnothing & \text { if } F[g]-K<-k, \\ \{p\} & \text { if } F[g]-K \geq-k,\end{cases}
$$

where $K=\mathrm{nl} b$ for all $b$ in $\operatorname{br} g$. 
We divide the proof into cases depending on the values of $m$ and $K$.

Case 1. The value $K$ is finite and the integer $m$ is strictly positive. By Proposition 3.2 we have

$$
0=A_{b(\mathrm{nl} \beta+m), \beta(\mathrm{nl} \beta)}=A_{b(\mathrm{nl} \beta+m+1), \beta(\mathrm{nl} \beta+1)}=\cdots=A_{b(P), \beta(P-m)}
$$

for all $b$ and $\beta$ in br $g$ such that $\mathrm{nl} \beta \leq P-m$. Thus, $A \equiv 0$ and $A$ is in the span of $M$.

Case 2. The integer $m$ is less than or equal to zero, or $K$ is infinite and $m$ is positive. In this case $\mathrm{nl} \beta \leq P-m$ for all $b$ and $\beta$ in br $g$ and the set of entries $A_{b(P), \beta(P-m)}$ can be index by $\mathbf{J} \times \mathbf{J}$. For each $(s, t)$ in $\mathbf{J} \times \mathbf{J}$ define $\lambda_{\mathrm{st}}: \mathbf{Z} \rightarrow \mathbf{Z}$ by

$$
\lambda_{\mathrm{st}}\left(b_{y}(x)\right)= \begin{cases}b_{z}(x-m) & \text { if } y \neq s \text { where } p=\left[\min [g]\left(b_{y} \cap b_{s}\right)\right]-F[g] \\ & \text { and } z=\min _{t, p-m}, \\ b_{t}(x-m) & \text { if } y=s,\end{cases}
$$

for $y$ in $\mathbf{J}$ and $x$ in $\mathbf{Z}$. The following two lemmas show that $T_{\lambda_{\text {st }}}$ is in $M$ for all $(s, t)$ in $\mathbf{J} \times \mathbf{J}$.

Lemma 1. For each $(s, t)$ in $\mathbf{J} \times \mathbf{J}$ the function $\lambda_{\mathrm{st}}$ is well defined.

Proof. Suppose $(s, t)$ is in $\mathbf{J} \times \mathbf{J}, y_{1}$ is in $\mathbf{J}, y_{2}$ is in $\mathbf{J}$ and $x$ is in $\mathbf{Z}$ such that $b_{y_{1}}(x)=b_{y_{2}}(x)$. We need to show that $\lambda_{\mathrm{st}}\left(b_{y_{1}}(x)\right)=\lambda_{\mathrm{st}}\left(b_{y_{2}}(x)\right)$. Let $L_{1}=\left[\min [g]\left(b_{y_{1}} \cap b_{s}\right)\right]-F[g], L_{2}=\left[\min [g]\left(b_{y_{2}} \cap b_{s}\right)\right]-F[g], z_{1}=\min \varsigma_{t, L_{1}-m}$ and $z_{2}=\min \varsigma_{t, L_{2}-m}$. If $x<L_{1}+F[g]$ and $x<L_{2}+F[g]$ then

$$
b_{s}\left(L_{1}+F[g]\right)=b_{y_{1}}\left(L_{1}+F[g]\right)=b_{y_{2}}\left(L_{1}+F[g]\right)
$$

and

$$
b_{s}\left(L_{2}+F[g]\right)=b_{y_{2}}\left(L_{2}+F[g]\right)=b_{y_{1}}\left(L_{2}+F[g]\right)
$$

so $L_{1}+F[g] \geq L_{2}+F[g]$ and $L_{1}+F[g] \leq L_{2}+[g]$ implying $L_{1}=L_{2}$ and $z_{1}=z_{2} ;$ thus,

$$
\lambda_{\mathrm{st}}\left(b_{y_{1}}(x)\right)=b_{z_{1}}(x-m)=b_{z_{2}}(x-m)=\lambda_{\mathrm{st}}\left(b_{y_{2}}(x)\right) .
$$

If $x \geq L_{1}+F[g]$ or $x \geq L_{2}+F[g]$ then $b_{y_{1}}(x)=b_{s}(x)=b_{y_{2}}(x)$ so $x \geq$ $L_{1}+F[g]$ and $x \geq L_{2}+F[g]$ implying $x-m \geq L_{1}-m+F[g]$ and $x-m \geq$ $L_{2}-m+F[g] ;$ thus, $z_{1}$ and $z_{2}$ are in $c_{t, x-m}$ and

$$
\lambda_{\mathrm{st}}\left(b_{y_{1}}(x)\right)=b_{z_{1}}(x-m)=b_{z_{2}}(x-m)=\lambda_{\mathrm{st}}\left(b_{y_{2}}(x)\right) \text {. }
$$

Lemma 2. For each $(s, t)$ in $\mathbf{J} \times \mathbf{J} \lambda_{\mathrm{st}} \circ g=g \circ \lambda_{\mathrm{st}}$.

Proof. Let $k$ be in $\mathbf{Z}$; then $k=b_{y}(x)$ for some $y$ in $\mathbf{J}$ and $x$ in $\mathbf{Z}$. Now

$$
\begin{aligned}
\lambda_{\mathrm{st}} \circ g(k) & =\lambda_{\mathrm{st}}\left(g\left(b_{y}(x)\right)\right)=\lambda_{\mathrm{st}}\left(b_{y}(x+1)\right)=b_{z}(x+1-m) \\
& =g\left(b_{z}(x-m)\right)=g\left(\lambda_{\mathrm{st}}\left(b_{y}(x)\right)\right)=g \circ \lambda_{\mathrm{st}}(k),
\end{aligned}
$$

where $z=\min \varsigma_{t, p-m}$ and $p=\left[\min [g]\left(b_{y} \cap b_{s}\right)\right]-F[g]$. 
In view of Lemmas 1 and 2 we need only show $A$ is a linear combination of operators of the form $T_{\lambda_{\text {st }}}$ where $(s, t)$ is in $\mathbf{J} \times \mathbf{J}$. To accomplish this, partition $\mathbf{J}$ into the subsets $O_{k} \stackrel{\lambda_{s l}}{=}\left\{t\right.$ in $\mathbf{J}: t \neq \min \varsigma_{t,-m+k-1}$ and $\left.t=\min \varsigma_{t,-m+k}\right\}$ for $k_{1} \leq k<k_{2}$, where $k_{1}=\max (0, m), k_{2}=\max (h, h+m)$ where $h=$ $\min \{q$ in $\mathbf{Z}:[g](q)=[g](r)$ implies $q=r\} ; O_{k_{2}}=\left\{t\right.$ in $\left.\mathbf{J}: t=\min _{t,-m+k_{2}}\right\}$ and $O_{k_{1}-1}=\varnothing$. Let $B_{k_{1}-1}=A$ and, for $k_{1} \leq k \leq k_{2}$, let

$$
B_{k}=B_{k-1}-\sum_{\substack{t \in O_{k} \\ s=\min \mathrm{\xi}_{s, k}}}\left(B_{k-1}\right)_{b_{s}(P), b_{t}(P-m)} T_{\lambda_{\mathrm{sl}}} .
$$

We now need to show that $B_{k_{2}}$ is the zero operator; then $A$ will clearly be in the span of $M$. By virtue of Proposition 3.3, this amounts to showing that $\left(B_{k_{2}}\right)_{b_{s}(P), b_{t}(P-m)}=0$ for all $(s, t)$ in $\mathbf{J} \times \mathbf{J}$.

We proceed inductively. Our inductive hypothesis is

$$
\left(B_{k}\right)_{b_{q}(P), b_{r}(P-m)}=0 \text { if } q \in \mathbf{J} \text { and } r \in \bigcup_{i=k_{1}-1}^{k} O_{i} .
$$

The induction hypothesis is trivially true for $k=k_{1}-1$ since $\bigcup_{i=k_{1}-1}^{k_{1}-1} O_{i}$ is the empty set.

Now suppose that for some $k$, where $k_{1}-1 \leq k<k_{2},\left(B_{k}\right)_{b_{q}(P), b_{r}(P-m)}=0$ when $q \in \mathbf{J}$ and $r \in \bigcup_{i=k_{1}-1}^{k} O_{i}$. We will demonstrate that $\left(B_{k+1}\right)_{b_{q}(P), b_{r}(P-m)}=$ 0 when $q \in \mathbf{J}$ and $r \in \bigcup_{i=k_{1}-1}^{k+1} O_{i}$.

If $t$ in $\mathbf{J}$ such that $t=\min \varsigma_{t,-m+k+1}, s$ in $\mathbf{J}$ such that $s=\min \varsigma_{s, k+1}$ and $q$ in $\mathrm{f}_{s, k+1}$ then using Proposition 3.2 and the inductive hypothesis we see that

$$
\begin{aligned}
& \left(B_{k}\right)_{b_{q}(P), b_{t}(P-m)}=\left(B_{k}\right)_{b_{q}(P), b_{t}(P-m)}+0 \\
& =\left(B_{k}\right)_{b_{q}(P), b_{t}(P-m)}+\sum_{\substack{r \in \mathrm{c}_{t,-m+k+1} \\
r \neq t}}\left(B_{k}\right)_{b_{q}(P), b_{r}(P-m)} \\
& =\sum_{r \in \mathrm{C}_{t,-m+k+1}}\left(B_{k}\right)_{b_{q}(P), b_{r}(P-m)}=\sum_{r \in \mathrm{\complement}_{t,-m+k+1}}\left(B_{k}\right)_{b_{q}(P+1), b_{r}(P-m+1)} \\
& =\cdots=\sum_{r \in \complement_{t,-m+k+1}}\left(B_{k}\right)_{b_{q}(F[g]+m), b_{r}(F[g])} \\
& =\sum_{\substack{r \in \mathrm{C}_{t,-m+k+1} \\
r=\min \boldsymbol{c}_{r, 1}}}\left(B_{k}\right)_{b_{q}(F[g]+m+1), b_{r}(F[g]+1)} \\
& =\sum_{\substack{r \in \mathcal{C}_{t,-m+k+1} \\
r=\min }}\left(B_{k}\right)_{b_{q}(F[g]+m+2), b_{r}(F[g]+2)} \\
& =\cdots=\left(B_{k}\right)_{b_{q}(F[g]+k+1), b_{t}(F[g]-m+k+1)}
\end{aligned}
$$

since $t=\min \mathrm{c}_{t,-m+k+1}$. Hence, $\left(B_{k}\right)_{b_{q}(P), b_{r}(P-m)}=\left(B_{k}\right)_{b_{x}(P), b_{t}(P-m)}$ for $s=$ $\min \varsigma_{s, k+1}, q$ and $x$ in $\varsigma_{s, k+1}$ and $t$ in $O_{k+1}$. 
Let $q, r, s, t \in \mathbf{J}$ and $y=\min [g]\left(b_{q} \cap b_{s}\right)-F[g]$. Then

$$
\begin{aligned}
& \left(T_{\lambda_{s 1}}\right)_{b_{q}(P), b_{r}(P-m)} \\
& =\frac{1}{\mu\left(b_{q}(P)\right)}\left\langle T_{\lambda_{\mathrm{sl}}} e_{b_{r}(P-m)}, e_{b_{q}(P)}\right\rangle \\
& =\frac{1}{\mu\left(b_{q}(P)\right)}\left\langle e_{b_{r}(P-m)}, T_{\lambda_{\mathrm{sl}}}^{*} e_{b_{q}(P)}\right\rangle \\
& =\frac{1}{\mu\left(\lambda_{\mathrm{st}}\left(b_{q}(P)\right)\right)}\left\langle e_{b_{r}(P-m)}, e_{\lambda_{\mathrm{sl}}\left(b_{q}(P)\right)}\right\rangle \\
& = \begin{cases}\frac{1}{\mu\left(b_{t}(P-m)\right)}\left\langle e_{b_{r}(P-m)}, e_{b_{t}(P-m)}\right\rangle & \text { if } q=s, \\
\frac{1}{\mu\left(b_{z}(P-m)\right.}\left\langle e_{b_{r}(P-m)}, e_{b_{z}(P-m)}\right\rangle & \text { if } q \neq s, \text { where } z=\min c_{t, y-m},\end{cases} \\
& = \begin{cases}0 & \text { if } q=s \text { and } r \neq t, \\
1 & \text { if } q=s \text { and } r=t, \\
0 & \text { if } q \neq s \text { and } r \neq \min c_{t, y-m}, \\
1 & \text { if } q \neq s \text { and } r=\min _{t, y-m} .\end{cases}
\end{aligned}
$$

Thus,

$$
\begin{aligned}
& \left(B_{k}\right)_{b_{s}(P), b_{t}(P-m)}\left(T_{\lambda_{\mathrm{sl}}}\right)_{b_{q}(P), b_{r}(P-m)} \\
& = \begin{cases}\left(B_{k}\right)_{b_{s}(P), b_{t}(P-m)} & \text { if }(q, r)=(s, t) \text { or } q \neq s \\
0 & \text { and } r=\min c_{t, y-m}, \\
0 & \text { otherwise. }\end{cases}
\end{aligned}
$$

If $r \in \bigcup_{i=k_{1}-1}^{k} O_{i}$ then $r \neq t$ and $r \neq \min \varsigma_{t, y-m}$, since $t \in O_{k+1}$. So

$$
\left(B_{k}\right)_{b_{s}(P), b_{t}(P-m)}\left(T_{\lambda_{\mathrm{st}}}\right)_{b_{q}(P), b_{r}(P-m)}=0 .
$$

If $r \in O_{k+1}$ then

$$
\begin{aligned}
& \left(B_{k}\right)_{b_{s}(P), b_{t}(P-m)}\left(T_{\lambda_{\mathrm{sl}}}\right)_{b_{q}(P), b_{r}(P-m)} \\
& = \begin{cases}\left(B_{k}\right)_{b_{q}(P), b_{r}(P-m)} & \text { if } r=t \text { and } q=s, \\
\left(B_{k}\right)_{b_{s}(P), b_{l}(P-m)} & \text { if } r=t, s=\min \varsigma_{q, k+1} \neq q, \\
0 & \text { otherwise, }\end{cases} \\
& = \begin{cases}\left(B_{k}\right)_{b_{q}(P), b_{r}(P-m)} & \text { if } r=t \text { and } s=\min \varsigma_{q, k+1}, \\
0 & \text { otherwise. }\end{cases}
\end{aligned}
$$

Let $r$ be in $\bigcup_{i=k_{1}-1}^{k} O_{i}$ and $q$ be in $\mathbf{J}$. Then

$$
\left(B_{k}-\sum_{\substack{t \in O_{k+1} \\ s=\min \mathcal{S}_{s, k+1}}}\left(B_{k}\right)_{b_{s}(P), b_{t}(P-m)} T_{\lambda_{\mathrm{sl}}}\right)_{b_{q}(P), b_{r}(P-m)}=\left(B_{k}\right)_{b_{q}(P), b_{r}(P-m)}-0=0
$$


TABLE 1

\begin{tabular}{l|l|l|l|l|l|l|l|l}
\hline Spaces & & $\cdots$ & $S_{-3}^{g}$ & $S_{-2}^{g}$ & $S_{-1}^{g}$ & $S_{0}^{g}$ & $S_{1}^{g}$ & $\ldots$ \\
\hline & $\begin{array}{l}\text { Basis } \\
\text { Elements }\end{array}$ & $\cdots$ & $e_{-6} e_{-5}$ & $e_{-4} e_{-3}$ & $e_{-2} e_{-1}$ & $e_{0}$ & $e_{1}$ & $\ldots$ \\
\hline
\end{tabular}

\begin{tabular}{c|c|c|c|c|c|c|c|c}
$\vdots$ & $\vdots$ & $\ddots$ & & & & & & \\
\hline$S_{-2}^{g}$ & $e_{-4}$ & & $A_{-4-6} A_{-4-5}$ & $A_{-4-4} A_{-4-3}$ & $A_{-4-2} A_{-4-1}$ & $A_{-40}$ & $A_{-41}$ & \\
& $e_{-3}$ & & $A_{-3-6} A_{-3-5}$ & $A_{-3-4} A_{-3-3}$ & $A_{-3-2} A_{-3-1}$ & $A_{-30}$ & $A_{-31}$ & \\
\hline$S_{-1}^{g}$ & $e_{-2}$ & & $A_{-2-6} A_{-2-5}$ & $A_{-2-4} A_{-2-3}$ & $A_{-2-2} A_{-2-1}$ & $A_{-20}$ & $A_{-21}$ & \\
\hline$S_{0}^{g}$ & $e_{0}$ & & $A_{-1-6} A_{-1-5}$ & $A_{-1-4} A_{-1-3}$ & $A_{-1-2} A_{-1-1}$ & $A_{-1}$ & $A_{-11}$ & \\
\hline$S_{1}^{g}$ & $e_{1}$ & & $A_{0-6} A_{0-5}$ & $A_{0-4} A_{0-3}$ & $A_{0-2} A_{0-1}$ & $A_{00}$ & $A_{01}$ & \\
\hline$\vdots$ & $\vdots$ & & & $A_{1-4} A_{1-3}$ & $A_{1-2} A_{1-1}$ & $A_{10}$ & $A_{11}$ & \\
\hline
\end{tabular}

by the induction hypothesis. Let $r$ be in $O_{k+1}$ and $q$ be in $\mathbf{J}$. Then

$$
\begin{gathered}
\left(B_{k}-\sum_{\substack{t \in O_{k+1} \\
s=\min \mathcal{C}_{s, k+1}}}\left(B_{k}\right)_{b_{s}(P), b_{t}(P-m)} T_{\lambda_{s t}}\right)_{b_{q}(P), b_{r}(P-m)} \\
=\left(B_{k}\right)_{b_{q}(P), b_{r}(P-m)}-\sum_{\substack{t=r \\
s=\min \mathcal{C}_{q, k+1}}}\left(B_{k}\right)_{b_{s}(P), b_{t}(P-m)} \\
=\left(B_{k}\right)_{b_{q}(P), b_{r}(P-m)}-\left(B_{k}\right)_{b_{q}(P), b_{r}(P-m)}=0 .
\end{gathered}
$$

Hence,

$$
\begin{aligned}
& \left(B_{k+1}\right)_{b_{q}(P), b_{r}(P-m)} \\
& =\left(B_{k}-\sum_{\substack{t \in O_{k+1} \\
s=\min \mathcal{C}_{s, k+1}}}\left(B_{k}\right)_{b_{s}(P), b_{s}(P-m)} T_{\lambda_{\mathrm{st}}}\right)_{b_{q}(P), b_{r}(P-m)}=0
\end{aligned}
$$

if $r$ is in $\bigcup_{i-k_{1}-1}^{k+1} O_{i}$ and $q$ is in $\mathbf{J}$. Therefore $\left(B_{k_{2}}\right)_{b_{q}(P), b_{r}(P-m)}=0$ for all $q$ and $r$ in $\mathbf{J}$. $\square$

The results of Theorem 3.4 can be extended to all operators in the commutant of $T_{g}$. Denote the set of bounded operators on $L^{2}(\mathbf{Z}, \mu)$ by $B\left(L^{2}(\mathbf{Z}, \mu)\right)$. For $A$ in $B\left(L^{2}(\mathbf{Z}, \mu)\right)$ with matrix $\left(A_{i j}\right)_{i, j \in \mathbf{Z}}$ and $m$ in $\mathbf{Z}$ define $D(A, m)$ on $L^{2}(\mathbf{Z}, \mu)$ to be the operator with matrix $\left(D(A, m)_{i, j}\right)_{i, j \in \mathbf{Z}}$ given by

$$
D(A, m)_{i, j}= \begin{cases}A_{i j} & \text { if }[g](i)=[g](j)+m \\ 0 & \text { otherwise. }\end{cases}
$$


TABLE 2

\begin{tabular}{l|l|l|l|l|l|l|l|l}
\hline Spaces & & $\cdots$ & $S_{-3}^{g}$ & $S_{-2}^{g}$ & $S_{-1}^{g}$ & $S_{0}^{g}$ & $S_{1}^{g}$ & $\cdots$ \\
\hline & $\begin{array}{l}\text { Basis } \\
\text { Elements }\end{array}$ & $\cdots$ & $e_{-6} e_{-5}$ & $e_{-4} e_{-3}$ & $e_{-2} e_{-1}$ & $e_{0}$ & $e_{1}$ & $\cdots$ \\
& & & & & & & \\
\hline
\end{tabular}

\begin{tabular}{|c|c|c|c|c|c|c|}
\hline$S_{-2}^{g}$ & $\begin{array}{l}e_{-4} \\
e_{-3}\end{array}$ & $\begin{array}{l}A_{-4-6} A_{-4-5} \\
A_{-3-6} A_{-3-5}\end{array}$ & $\begin{array}{ll}0 & 0 \\
0 & 0\end{array}$ & $\begin{array}{ll}0 & 0 \\
0 & 0\end{array}$ & $\begin{array}{l}0 \\
0\end{array}$ & $\begin{array}{l}0 \\
0\end{array}$ \\
\hline$S_{-1}^{g}$ & $\begin{array}{l}e_{-2} \\
e_{-1}\end{array}$ & $\begin{array}{ll}0 & 0 \\
0 & 0\end{array}$ & $\begin{array}{l}A_{-2-4} A_{-2-3} \\
A_{-1-4} A_{-1-3}\end{array}$ & $\begin{array}{ll}0 & 0 \\
0 & 0\end{array}$ & $\begin{array}{l}0 \\
0\end{array}$ & $\begin{array}{l}0 \\
0\end{array}$ \\
\hline$S_{0}^{g}$ & $e_{0}$ & 00 & 00 & $A_{0-2} A_{0-1}$ & 0 & 0 \\
\hline$S_{1}^{g}$ & $e_{1}$ & 00 & 00 & 00 & $A_{10}$ & 0 \\
\hline$\vdots$ & $\vdots$ & & & & & \\
\hline
\end{tabular}

The operators $D(A, m)$ are in $B\left(L^{2}(\mathbf{Z}, \mu)\right)$ and $A$ is the weak operator limit of $A_{n}=\sum_{m=-n}^{n} D(A, m)$ as $n$ goes to $\infty$.

Example 6. Let $g: \mathbf{Z} \rightarrow \mathbf{Z}$ be the function that has the graph:

$$
\begin{aligned}
& \cdots \rightarrow-3 \rightarrow-1 \\
& \\
& \cdots \rightarrow-4 \rightarrow-2
\end{aligned}
$$

Without loss of generality, let $[g](0)=0$. Then $S_{m}^{g}=\operatorname{span}\left\{e_{-2 m}, e_{-2 m+1}\right\}$ when $m<0$ and $S_{m}^{g}=\operatorname{span}\left\{e_{m}\right\}$ and $m \geq 0$. Suppose that $A$ is an operator that commutes with $T_{g}$. Table 1 illustrates the matrix for $A$ indexed in relation to the spaces $S_{m}^{g}$ and also to the basis elements $e_{m}$.

An operator $D(A, m)$ is an operator that has zero entries except for one matrix diagonal (indexed by the spaces $S_{i}$ ). Table 2 illustrates $D(A, 1)$. Note that

$$
\left(\begin{array}{ll}
A_{-2,-4} & A_{-2,-3} \\
A_{-1,-4} & A_{-1,-3}
\end{array}\right)
$$

are the matrix entries in Proposition 3.3.

Theorem 3.5. Let $g: \mathbf{Z} \rightarrow \mathbf{Z}$ have one orbit, no cycle, more than one branch, and only a finite number of branches. In addition assume there is $K$ in $\mathbf{Z} \cup\{-\infty\}$ such that $\mathrm{nl} b=k$ for all $b$ in br $g$. Then in the space $L^{2}(\mathbf{Z}, \mu)$,

$$
\left\{T_{g}\right\}^{\prime}=\left[B\left(L^{2}(\mathbf{Z}, \mu)\right) \cap \operatorname{span}\left\{T_{x}: X \circ g=g \circ X, X: \mathbf{Z} \rightarrow \mathbf{Z}\right\}\right] .
$$


Proof. Let $A$ be in $\left\{T_{g}\right\}^{\prime}$ and $m$ be in $\mathbf{Z}$. The operator $D(A, m)$ satisfies Proposition 3.2 and therefore $D(A, m)$ is in $\left\{T_{g}\right\}^{\prime}$. Theorem 3.4 implies $D(A, m)$ is in the span of $\left\{T_{x}: X \circ g=g \circ X, X: \mathbf{Z} \rightarrow \mathbf{Z}\right\}$. Hence, for any positive integer $n, A_{n}$ is in the span of $\left\{T_{x}: X \circ g=g \circ X, X: \mathbf{Z} \rightarrow \mathbf{Z}\right\}$. Conversely, it is clear that $B\left(L^{2}(\mathbf{Z}, \mu)\right) \cap \operatorname{span}\left\{T_{x}: X \circ g=g \circ X, X: \mathbf{Z} \rightarrow \mathbf{Z}\right\}$ is contained in $\left\{T_{g}\right\}^{\prime}$.

Theorem 3.5 leaves us with some unanswered questions. Is it possible to choose functions that commute with $T_{g}$ in such a way that the commutant of $T_{g}$ is the weak closure of the span of $\left\{T_{x}: X \circ g=g \circ X\right.$ and $T_{x}$ is bounded $\}$ or maybe even that the commutant of $T_{g}$ is the span of $\left\{T_{x}: X \circ g=g \circ X\right.$ and $T_{x}$ is bounded ? What should the conclusion of Theorem 3.5 be if the branches of $g$ are allowed to have different negative lengths? In this case the functions $\lambda_{\text {st }}$ constructed in Theorem 3.4 may not commute with $g$, so that an easy modification of Theorem 3.4 is not possible.

\section{BIBLIOGRAPHY}

1. J. W. Carlson, Weighted composition operators on $l^{2}$, Dissertation, Purdue University, West Lafayette, Indiana, 1985.

2. J. R. Choksi, Unitary operators induced by measure preserving transformations, J. Math. Mech. (Indiana Univ. Math J.) 16 (1966), 83-100.

3. __ Unitary operators induced by measurable transformations, J. Math. Mech. (Indiana Univ. Math J.) 17 (1968), 781-801.

4. J. A. Cima, J. E. Thompson, and W. R. Wogen, On some properties of composition operators, Indiana Univ. Math. J. 24 (1974), 215-220.

5. J. A. Cima and W. R. Wogen, On algebras generated by composition operators, Canad. J. Math. 26 (1974), 1234-1241.

6. J. B. Conway, Subnormal operators, Pitman, London, 1981.

7. C. C. Cowen, Composition operators on $H^{2}$, J. Operator Theory 9 (1983), 77-106.

8. J. A. Deddens, Analytic Toeplitz and composition operators, Canad. J. Math. 24 (1972), 859865.

9. M. Denker, On unitary operators inducing measure-preserving transformation, Math. Z. 160 (1978), 163-172.

10. D. K. Gupta, A. Kumar, and R. K. Singh, Quasinormal composition operators on $L_{p}^{2}$, Indian J. Pure Appl. Math. 11 (1980), 904-907.

11. P. R. Halmos, A Hilbert space problem book, Van Nostrand, Princeton, N. J., 1967.

12. A. Iwanik, Pointwise induced operators on $L^{p}$-spaces, Proc. Amer. Math. Soc. 58 (1976), 173178.

13. R. L. Kelley, Weighted shifts on Hilbert space, Dissertation, University of Michigan, Ann Arbor, 1966.

14. B. S. Komal and R. K. Singh, Composition operators on $l^{p}$ and its adjoint, Proc. Amer. Math. Soc. 70 (1978), 21-25.

15. A. Kumar, Fredholm composition operators, Proc. Amer. Math. Soc. 79 (1980), 233-236.

16. A. Kumar and R.K. Singh, Multiplication operators and composition operators with closed ranges, Bull. Austral. Math. Soc. 16 (1977), 247-252.

17. D. E. Marshall and K. Shephensen, Inner divisors and composition operators, J. Funct. Anal. 46 (1982), 131-148. 
18. D. H. Mayer, On composition operators on Banach spaces of holomorphic functions, J. Funct. Anal. 35 (1980), 191-206.

19. N. K. Nikolskii, On invariant subspaces of weighted shift operators, Math. USSR Sb. 3 (1967), 159-176.

20. E. A. Nordgren, Composition operators, Canad. J. Math. 20 (1968), 442-449.

21. __ Composition operators on Hilbert spaces, Hilbert Space Operators, Lecture Notes in Math., vol. 693, Springer-Verlag, Berlin, 1978, pp. 37-63.

22. S. K. Parrott, Weighted translation operators, Thesis, University of Michigan, Ann Arbor, 1965.

23. W. C. Ridge, Spectrum of a composition operator, Proc. Amer. Math. Soc. 37 (1973), 121-127.

24. Characterization of abstract composition operators, Proc. Amer. Math. Soc. 45 (1974), 393-396.

25. A. L. Shields, Weighted shift operators and analytic function theory, Topics in Operator Theory (C. Pearcy, ed.), Math. Surveys, no. 13, Amer. Math. Soc., Providence, R. I., 1974, pp. 49-128.

26. R. K. Singh, Compact and quasinormal composition operators, Proc. Amer. Math. Soc. 45 (1974), 80-82.

27. $\ldots$, Normal and hermitian composition operators, Proc. Amer. Math. Soc. 47 (1975), 348350.

28. __ Invertible composition operators on $L^{2}(\lambda)$, Proc. Amer. Math. Soc. 56 (1976), 127-129.

29. __ Composition operators induced by rational functions, Proc. Amer. Math. Soc. 59 (1976), 329-333.

30. __ Inner functions and composition operators of a Hardy space, Indian J. Pure Appl. Math. 11 (1980), 1297-1300.

31. __, Quasinormal composition operators, Indian J. Pure Appl. Math. 13 (1982), 8-12.

32. A. E. Taylor and D. C. Lay, Introduction to functional analysis, 2nd ed., Wiley, New York, 1980.

33. R. Whitley, Normal and quasinormal composition operators, Proc. Amer. Math. Soc. 70 (1978), 114-118.

Department of Mathematics, Creighton University, Omaha, Nebraska 68178-0109 